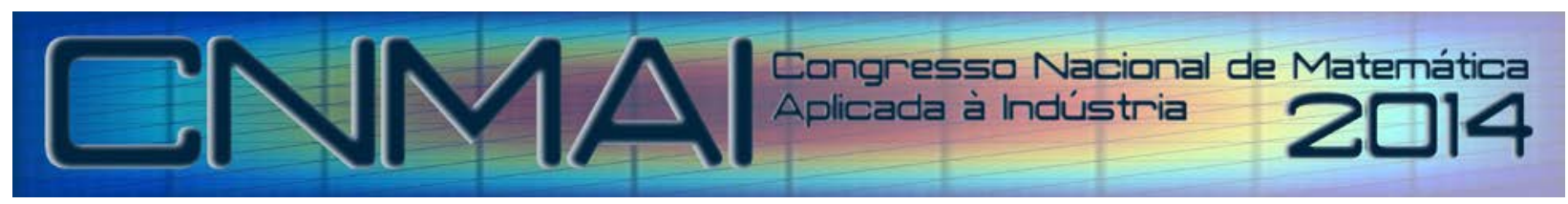

18 a 21 de novembro de 2014, Caldas Novas - Goiás

\title{
UTILIZAÇÃO DE UMA MALHA ESTRUTURADA NA ANÁLISE DO AEROFÓLIO S809
}

\author{
Wander Martins Ribeiro, wander.ribeiro@ufv.br ${ }^{1}$ \\ Julio Cesar Costa campos, julio.campos@ufv.br ${ }^{2}$ \\ Álvaro M. Bigonha Tibiriça, alvaro.tibirica@ufv.br ${ }^{3}$ \\ Henrique Marcio Pereira Rosa, henrique.rosa@ufv.br ${ }^{4}$ \\ 1,2,3,4 Universidade Federal de Viçosa - Departamento de Engenharia de produção e Mecânica. Rua PH Rolfs s/n. \\ Rogério Fernandes Brito, rogbrito@unifei.edu.br ${ }^{5}$ \\ ${ }^{5}$ Universidade Federal de Itajubá - Campus Itabira. Rua Irmã Ivone Drumond, 200, Distrito Industrial II. \\ Pedro Casanova Treto, pcasanova2000@gmail.com ${ }^{6}$ \\ ${ }^{6}$ Universidad de Costa Rica - Instituto de Investigaciones en Ingeniería - INII. San Pedro de Montes de Oca.
}

\begin{abstract}
Resumo: A análise numérica tem se tornado uma ferramenta essencial nas etapas do desenvolvimento de um projeto devido a sua capacidade de solução para fenômenos físicos descritos por leis de difícil solução analítica, economizando tempo e dinheiro, uma vez que um número muito menor de protótipos será construído até a obtenção do resultado final. Para tanto, deve-se encontrar uma malha ideal de forma que esta possibilite o resultado mais próximo possível da realidade, com o mínimo de esforço computacional. O objetivo é o estudo do aerofólio S809 bastante empregado em aerogeradores. A metodologia empregada será a determinação de uma malha estruturada ideal para simulações em diversas condições de operação dos rotores desses equipamentos. Será observado alguns aspectos importantes, como o número mínimo de elementos necessários, o refinamento próximo à superfície do aerofólio, isto é, o tamanho inicial e a relação de crescimento dos elementos nesta região, de maneira a obter a melhor representação dos efeitos de camada limite e o fenômeno da turbulência, assim como, o tamanho mínimo necessário do domínio a ser discretizado, de forma que este não influencie nos resultados das simulações. Desta forma, aplicando-se o aumento gradativo do número de elementos bem como a diminuição da relação de crescimento destes ao redor do aerofólio nas malhas construídas pelo software ICEM® e realizando simulações através do FLUENT®, espera-se a convergência dos resultados obtidos para os coeficientes de arrasto e de sustentação a partir de um determinado número de elementos da malha, sendo desnecessário qualquer tipo de acréscimo no refinamento local ou global, uma vez que isto apenas aumentaria o esforço computacional e não mais influenciaria nos resultados. Além disso, deve ser observado que as equações de momento, energia e da continuidade devem atender ao erro mínimo exigido. Assim, esta malha representaria o caso ideal desejado.
\end{abstract}

Palavras-chave: análise numérica, aerofólio S809, malha estruturada, Fluent

\section{INTRODUÇÃO}

A produção de energia elétrica através da força eólica iniciou-se, somente, por volta do século XX, com o crescimento da economia mundial, onde se teve o aumento significativo do consumo de eletricidade. Atualmente a produção de energia eólica é desejada por se tratar de uma fonte não poluente e teoricamente inesgotável. Outro fato que justifica a sua produção é o elevado apelo ecológico que se faz necessário diante de algumas circunstâncias..

As simulações numéricas são muito importantes, pois elas têm um enorme potencial para predizer o desempenho dos equipamentos e processos antes da sua produção e implementação, assim como permitir a redução do tempo de desenvolvimento do projeto de uma empresa. Mas, para a confiabilidade dos resultados os ensaios experimentais são fundamentais para a validação dos modelos testados. 
Com aumento da velocidade de processamento dos computadores e sua capacidade de armazenamento torna-se possível realizar análises numéricas de fenômenos físicos complexos. Neste estudo propõe-se a discutir a malha estruturada que melhor representa o fenômeno físico do aerofólio S809, o qual possa gerar resultados confiáveis com o “mundo real”. Logo, estes resultados devem expressar uma consistência física do fenômeno, de tal forma que seja seguro e preciso.

\section{MÉTODO COMPUTACIONAL}

Neste trabalho foi utilizado o código computacional ANSYS FLUENT para representar fisicamente o escoamento ao redor do aerofólio S809.

Neste código os volumes de controle são centrados na célula, enquanto que no código CFX os volumes de controle são centrados no nó.

O método computacional utilizado, neste código, é o método de volumes finitos.

\subsection{Geometria Utilizada}

A geometria do S809 foi construída graficamente com auxílio do software Solidworks. Após a execução desta geometria ela foi exportada para um gerador de malhas ICEM-CFD do pacote ANSYS. A Fig.1 evidencia a geometria do Aerofólio S809 elaborada no Solidworks. A geração de uma malha define em todo o domínio computacional as células nas quais as variáveis de escoamento, como por exemplo, velocidade, pressão são calculadas.

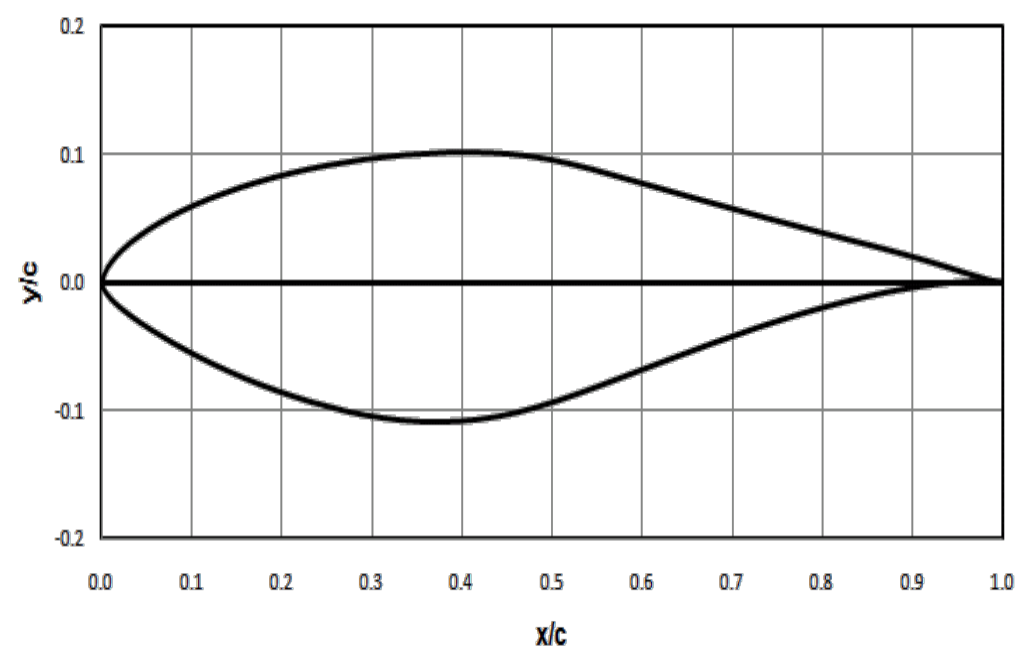

Figura 1. Representação do perfil do aerofólio S809, desenhado no Solidworks.

\subsection{Equações Governantes e Discretização}

As equações gerais de conservação, tais como, de massa, momentum, energia e entre outras foram resolvidas para um conjunto de volumes de controle.

O domínio do aerofólio S809 foi discretizado para esse conjunto de volumes de controle.

Utilizou-se o modelo de turbulência padrão do tipo k-E. Esse modelo é amplamente utilizado, como modelo de turbulência, em aplicações industriais.

\subsection{Condições de Contorno}

A condição de contorno apropriada se faz necessária para estabelecer uma solução CFD exata. A condição de contorno mais simples é a da parede. Neste caso o fluido não pode ultrapassar á parede, então a componente normal da velocidade é igual a zero, o que caracteriza uma condição de contorno de parede prescrita. Desta forma, as paredes foram consideradas adiabáticas e com a condição de não deslizamento.

Da mesma maneira, definiu-se as condições de contorno para escoamento de entrada e de saída para S809. Enfatiza-se, nesta situação, o fluido (ar) entra no domínio computacional e sai do domínio.

Neste trabalho, estabeleceu-se a velocidade de entrada, $30 \mathrm{~m} / \mathrm{s}$, como condição especificada de velocidade e pressão ambiente distante do aerofólio S809 como condição de pressão especificada. A pressão, próxima ao aerofólio S809, isto é, na parte superior e inferior foram calculadas. 


\subsection{Geração da Malha}

Utilizou-se o gerador de malhas ICEM do pacote ANSYS para gerar a malha do tipo C-H para o aerofólio S809.

É uma das principais etapas da simulação computacional, pois ela é que representará o seu domínio contínuo através de pontos interligados, onde serão resolvidas as equações governantes do seu fenômeno. A representação da malha estruturada do tipo C-H em 2D pode ser vista na Fig.2.

A malha consta de 1528900 elementos e para obter uma boa previsão do arrasto e do modelo de turbulência, a malha foi bem resolvida próximo a parede, isto é, o primeiro ponto do grid está localizado na subcamada viscosa, $\mathrm{y}^{+}<$ 10. A extensão do grid na direção radial foi de 20c, onde c representa o comprimento da corda, contado a partir do centro de gravidade do aerofólio S809.

A extensão de 20c se fez necessário para garantir que o domínio computacional seja suficientemente grande, de forma a não permitir escoamento reverso.

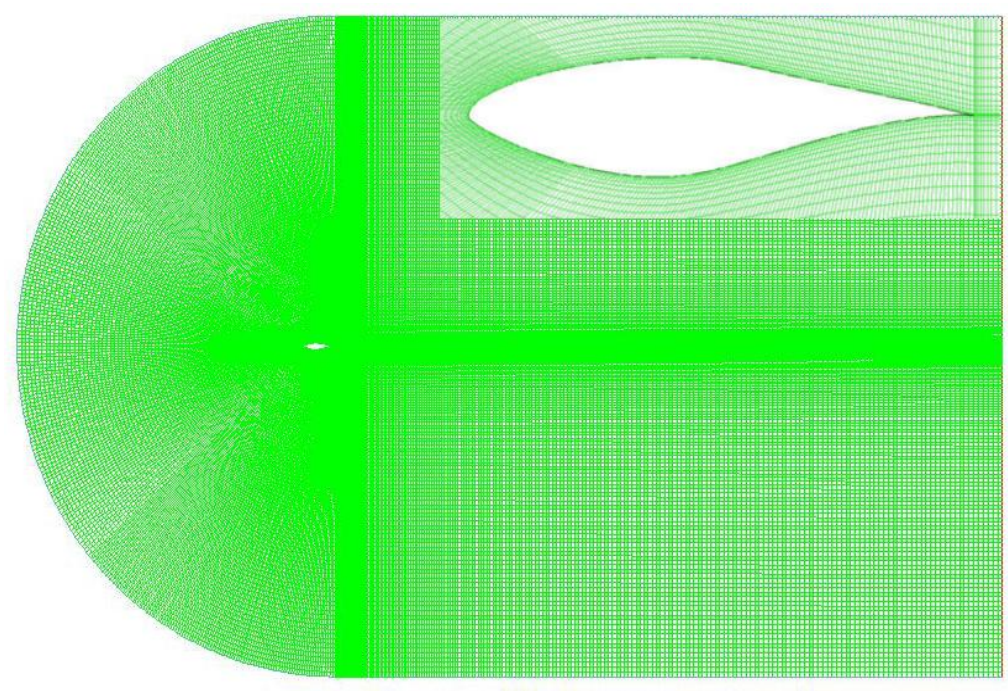

Figura 2. Representação da malha estruturada do tipo C-H, gerada a partir do ICEM.

Utilizou-se uma malha estruturada composta por células planares com quatro arestas ao redor do aerofólio S809, conforme evidenciado na Fig.3.

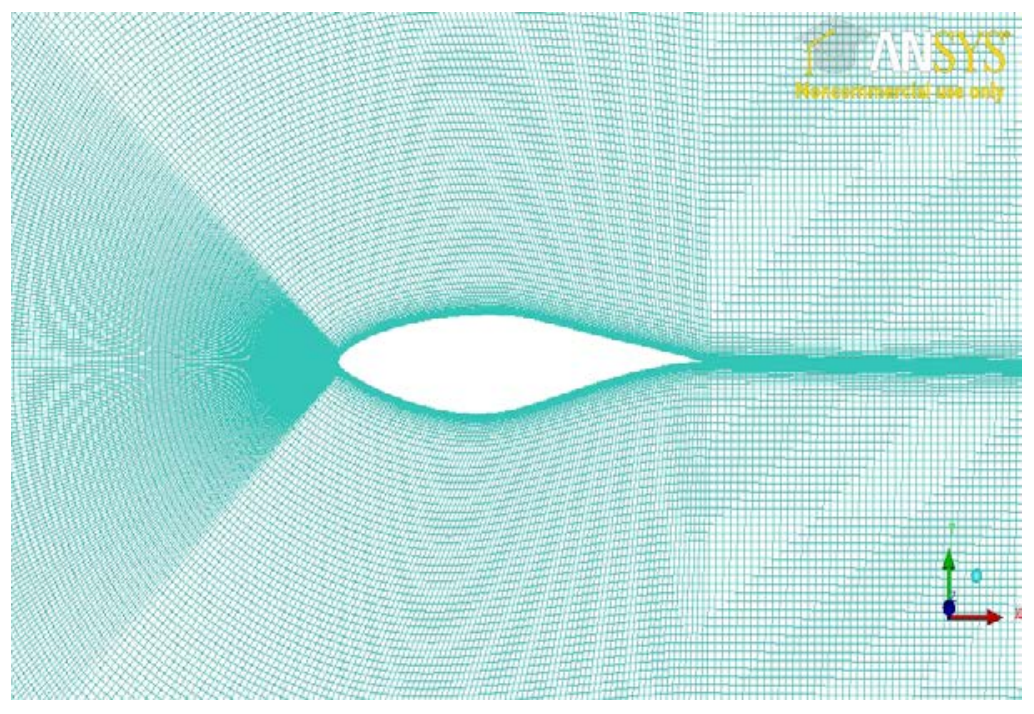

Figura 3. Representação da malha estruturada em torno do aerofólio S809.

Çengel (2007) levanta a discussão sobre malha estruturada e não estruturada, onde define-se a malha estruturada composta de células planares com quatro arestas em 2-D. De outra maneira, a malha não estruturada consiste de células em diversas formas, sendo que, na dimensão 2-D frequentemente usa-se triângulos ou quadriláteros. Ele enfatiza que a malha estruturada é recomendada para as camadas limites, onde as variáveis de escoamento mudam rapidamente na direção normal à parede, sendo que, malhas com alta resolução são necessárias próximas á parede. Nesta situação o refinamento da malha é importante junto às paredes já que nessas áreas a velocidade do escoamento é zero e as tensões 
cisalhantes são máximas. Portanto, nessa região, ocorre a predominância dos efeitos viscosos, onde se faz necessário os detalhes do escoamento. Nesta situação a malha estruturada permite uma resolução mais fina do que as malhas não estruturadas para o mesmo número de células.

A Figura 4 ilustra a malha estruturada para região de bordo de ataque do aerofólio S809.

Nesta Figura evidencia uma malha extremamente fina próxima ao bordo de ataque do aerofólio S809, objeto de estudo deste trabalho. Isto se fez necessário para resolver a camada limite, conforme enfatizado por Çengel (2007), no parágrafo anterior.

A confecção da malha permite a aplicação do método dos volumes finitos. Esse método consiste em dividir a geometria em pedaços menores, discretizando assim o volume contínuo do sólido. Cada pedacinho mantém as características originais, isso facilita o cálculo das equações diferenciais, tornando-as um sistema de equações algébricas e a definição das condições de contorno. Para se obter resultados mais precisos é necessário que a malha seja completamente compatível com o tipo de escoamento. Para isso os pequenos volumes devem ser menores em algumas regiões do que em outras para que possam captar os detalhes do escoamento, como alteração de velocidade, de pressão, de temperatura e formação de vórtices.

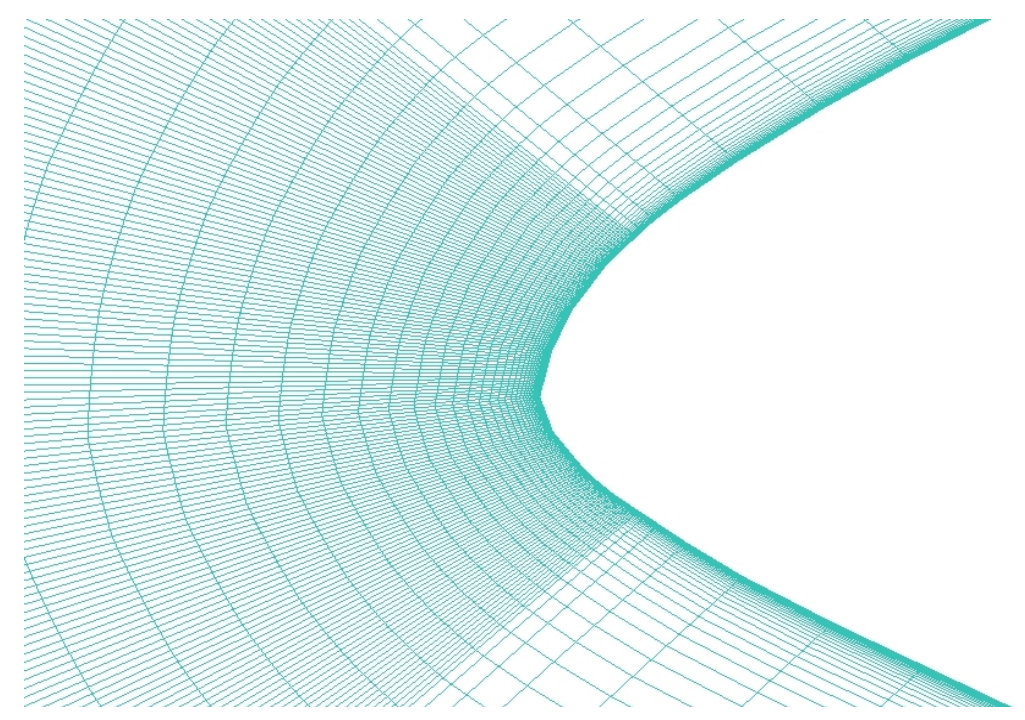

Figura 4. Representação da malha estruturada para o bordo de ataque do S809.

\section{RESULTADOS E CONCLUSÕES}

Devido a ação do vento sobre a superfície do perfil do aerofólio, as forças de pressão atuam normal a esta superfície e a força cortante atua de forma tangencial. Integrando estas forças que atuam sobre a superfície do aerofólio em estudo obtém-se uma força resultante e um momento. A força resultante pode ser dividida em duas componentes, sendo uma perpendicular a corda do perfil, força normal, e a outra paralela a corda do perfil, força axial. Estas forças podem ser determinadas durante a análise dos resultados no FLUENT, por meio do coeficiente axial, $\mathrm{C}_{\mathrm{A}}$, e normal, $\mathrm{C}_{\mathrm{N}}$, para os ângulos de ataque, $\alpha=0^{\circ}, 4^{\circ}, 8^{\circ}, 12^{\circ}, 16^{\circ}$ e $20^{\circ}$. TRETO, (2013), afirma que com estas informações determina-se os coeficientes de sustentação e arrasto representados na Eq. (1) e (2).

$$
\begin{aligned}
& C_{d}=C_{N} \operatorname{sen} \alpha-C_{A} \cos \alpha \\
& C_{l}=C_{N} \cos \alpha-C_{A} \operatorname{sen} \alpha
\end{aligned}
$$

As Figuras 5 e 6 representam os resultados numéricos para o coeficiente de arrasto e sustentação do perfil do aerofólio S809.

Conforme enfatizado, anteriormente, o modelo de turbulência utilizado neste artigo é o modelo k-ع. A partir das Figuras 5 e 6 verifica-se que os resultados reproduzem o processo físico em estudo.

Percebe-se nestas figuras que a curva apresentada tem o mesmo comportamento que as curvas apresentadas nos trabalhos de (Treto et al., 2013; Castelli et al., 2012; Wolfe et al., 1997; Yao et al., 2012; Ramsay et al., 1995).

Embora não foi feito um estudo comparativo com os resultados experimentais, as figuras apresentadas mostram uma boa precisão dos resultados do coeficiente de arrasto e sustentação.

A partir dos resultados apresentados nestas figuras ficou evidenciado que a malha utilizada nesta análise foi eficiente para representar o fenômeno físico.

Isto nos revela que as equações algébricas e a definição das condições de contorno são coerentes, para obter resultados mais precisos, desde que a malha seja completamente compatível com o tipo de escoamento. Para isso os 
pequenos volumes devem ser menores em algumas regiões do que em outras para que possam captar os detalhes do escoamento, como alteração de velocidade, de pressão, de temperatura e formação de vórtices.

Evidenciou-se na Fig. 5 que para número de Reynolds elevado, neste estudo, $\operatorname{Re}=2 \times 10^{6}$, o 0,027< $\mathrm{C}_{\mathrm{d}}<0,098$. De forma similar, na Fig. 6 o coeficiente de sustentação está na faixa de 0,094 $<\mathrm{C}_{\mathrm{l}}<0,92$.

Percebe-se na Fig. 6 que a partir de um ângulo de ataque de $12^{\circ}$ ocorre uma queda do coeficiente de sustentação. Isto representa o stol, caracterizando o descolamento da camada limite. Este comportamento, também, é evidenciado em vários trabalhos citados anteriormente, assim como, em Gross et al. (2011).

Concluo, enfatizando que o modelo de turbulência é importante na análise de escoamento de fluido ao redor de aerofólios, assim como, a caracterização da malha e seu refinamento. Portanto, deve-se simular outros tipos de modelos de turbulência para as devidas comparações.

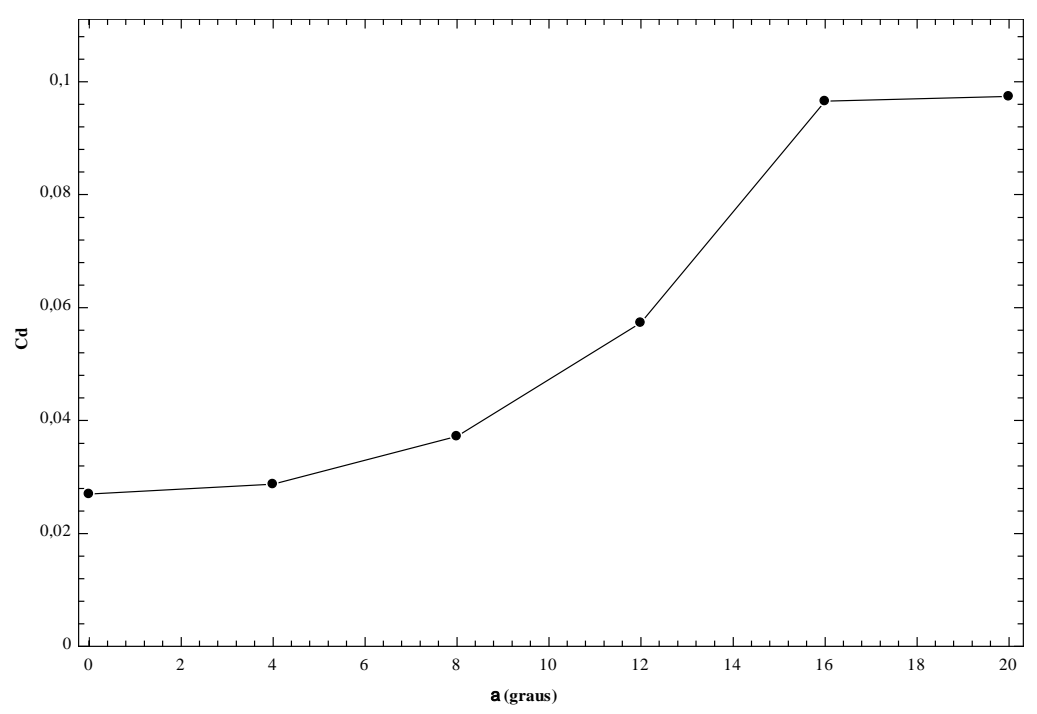

Figura 5. Comportamento do coeficiente de arrasto em função do ângulo de ataque.

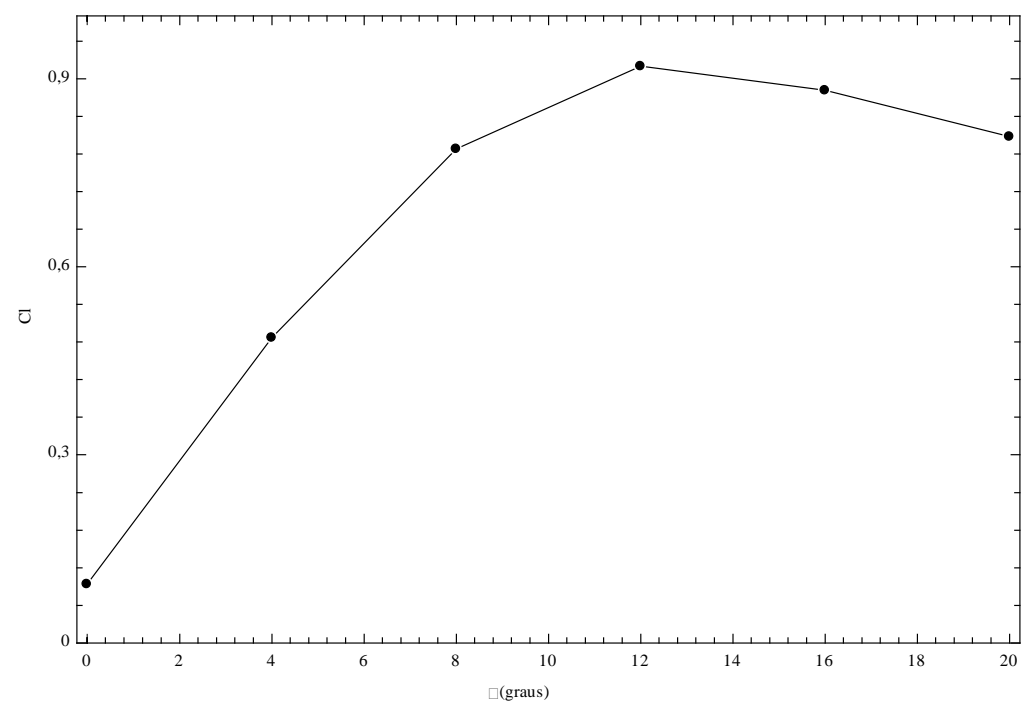

Figura 6. Comportamento do coeficiente de sustentação em função do ângulo de ataque.

\section{AGRADECIMENTOS}

Os autores agradecem a Fundação de Amparo à Pesquisa do Estado de Minas Gerais (FAPEMIG) e a Universidade Federal de Viçosa (UFV) pela realização deste trabalho.

\section{REFERÊNCIAS}

Castelli, E.B., Raciti, M., Grandi, G. 2012. Numerical Analysis of Laminar to Turbulent Transition on the DU91-W2250 airfoil, World Academy of Science, Engineering and Technology.

Çengel, Y. A., Cimbala, J.M. (2007). Mecânica dos Fluidos - Fundamentos e Aplicações. S. Paulo, Brazil: Ed. McgrawHill. 816 p. 
Gross, A. and Fasel, H.F. 2011. Numerical Investigation of Different Wind Turbine Airfoils. 49th AIAA Aerospace Sciences Meeting including the New Horizons Forum and Aerospace Exposition. Orlando, Florida.

Ramsay, R.F., Hoffman, M.J., Gregorek, G.M. 1995. Effects of grit roughness and pitch oscillations on the S809 airfoil, Golden, CO.

Treto, P. Casanova, Ramírez, K. Solís y Campos, Julio C. C. 2013. Comportamiento aerodinámico del perfil S809 para bajo número de Reynolds. Numerical Heat Transfer, 3(2), 149-167.

Yao, J., Yuan, W., Wang, J., Xie, J., Zhou, H., Peng, M., et al. 2012. Numerical simulation of aerodynamic performance for two dimensional wind turbine airfoils, Procedia Engineering. 31- 80-86.

Wolfe, W., Ochs, S. 1997. CFD calculations of S809 aerodynamic characteristics, in: 35th Aerospace Sciences Meeting and Exhibit, American Institute of Aeronautics and Astronautics, Reston, Virgina.

\title{
RESPONSABILIDADE AUTORAL
}

Os autores são os únicos responsáveis pelo conteúdo deste trabalho.

\section{THE USE Of A STRUCTURED MESH ANALYSIS Of AEROFOIL S809}

\author{
Wander Martins Ribeiro, wander.ribeiro@ufv.br ${ }^{1}$ \\ Julio Cesar Costa campos, julio.campos@ufv.br ${ }^{2}$ \\ Álvaro M. Bigonha Tibiriça, alvaro.tibirica@ufv.br ${ }^{3}$ \\ Henrique Marcio Pereira Rosa, henrique.rosa@ufv.br ${ }^{4}$ \\ 1,2,3,4 Universidade Federal de Viçosa - Departamento de Engenharia de produção e Mecânica. Rua PH Rolfs s/n. \\ Rogério Fernandes Brito, rogbrito@unifei.edu.br ${ }^{5}$ \\ ${ }^{5}$ Universidade Federal de Itajubá - Campus Itabira. Rua Irmã Ivone Drumond, 200, Distrito Industrial II. \\ Pedro Casanova Treto, pcasanova2000@gmail.com ${ }^{6}$ \\ ${ }^{6}$ Universidad de Costa Rica - Instituto de Investigaciones en Ingeniería - INII. San Pedro de Montes de Oca.
}

\begin{abstract}
The numerical analysis has become an essential tool in the steps of a project development due to its ability to solve physical phenomena described by laws with complex analytical solutions, saving time and money, once a much lower number of prototypes will be built until the achievement of the final result. However we must find an ideal mesh to enable a result as close as it is possible from reality, and with the less computational effort. The target is the study of the aerofoil S809 much used in aerogenerators. The methodology applied will be the determination of a structured mesh ideal for simulation in various conditions of operation for the rotors of these equipment. Some important aspects must be noticed, such as the less number of elements required, the refinement near the surface of the aerofoil, which is the initial size and the growth relation of the elements in this region, to obtain the best representation of the boundary layer effects and the phenomenon of turbulence, such as the minimum size necessary of the domain to be discretized, in a way that it does not interfere in the simulation results. Thus, applying the gradual increase of the number of elements as well as the decrease of the relation of growth between those around the aerofoil in the meshes built by the software ICEM® and performing the simulations using the FLUENT®, it is expected the convergence of the results obtained to the drag and lift coefficients from a determined number of elements of the mesh, being unnecessary any kind of addition in the local or global refinement, once it would only increase the computational effort and it would not influence the results no more. Besides, it must be observed that the momentum, energy, and continuity equations must attend to the minimum error required. Thus, this would represent the ideal case desired.
\end{abstract}

Keywords: numerical analysis, airfoil S809, structured mesh,Fluent

\section{RESPONSABILIDADE AUTORAL}

The author(s) is (are) the only responsible for the printed material included in this paper. 